\title{
MCP detector development for WSO-UV
}

\author{
Sebastian Diebold ${ }^{a}$, Jürgen Barnstedt ${ }^{a}$, Hans-Rudolf Elsener ${ }^{b}$, Philipp Ganz ${ }^{c}$, \\ Stephan Hermanutz ${ }^{a}$, Christoph Kalkuhl ${ }^{a}$, Norbert Kappelmann ${ }^{a}$, Marc Pfeifer ${ }^{a}$, \\ Daniel Schaadt ${ }^{d}$, Thomas Schanz ${ }^{a}$, Omar Tanirah ${ }^{c}$, Klaus Werner $^{a}$ \\ ${ }^{a}$ Institute for Astronomy and Astrophysics (IAAT), University of Tübingen, \\ Sand 1, Tübingen, Germany; \\ ${ }^{b}$ Empa, Swiss Federal Laboratories for Materials Science and Technology, \\ Überlandstrasse 129, Dübendorf, Switzerland; \\ ${ }^{c}$ Institute of Applied Physics, Karlsruhe Institute of Technology (KIT), \\ Wolfgang-Gaede-Str. 1a, Karlsruhe, Germany; \\ ${ }^{d}$ Institute of Energy Research and Physical Technologies, Clausthal University of Technology, \\ Leibnizstraße 4, Clausthal, Germany
}

\begin{abstract}
The spectrographs of WSO-UV cover the wavelength range of $102-310 \mathrm{~nm}$. The essential requirements for the associated detectors are high quantum efficiency, solar blindness, and single photon detection. To achieve this, we develop a microchannel plate detector in a sealed tube. We plan to use cesium activated gallium nitride as semitransparent photocathode, a stack of two microchannel plates and a cross strip anode with advanced readout electronics. Challenges are the degradation of the photocathode under atmospheric conditions and the sealing process. We present the detector concept, details of the transfer and sealing processes under UHV, and the current status.
\end{abstract}

Keywords: Photon detector, UV detector, MCP, sealed tube, indium sealing, WSO-UV

\section{INTRODUCTION}

\subsection{WSO-UV Mission Overview}

The World Space Observatory - Ultraviolet (WSO-UV) is an international astronomical observatory, designed to provide future access to the $100-300 \mathrm{~nm}$ spectral range. The importance of this mission gets obvious, regarding the major impact UV observations have had on modern astronomy together with the fact, that no other UV mission is planned for the near future, i.e. the post-HST era.

WSO-UV is a multinational project under Russian leadership with contributions from Spain and Ukraine. The Institute for Astronomy and Astrophysics of the University of Tübingen (IAAT) will provide detectors for this mission. In combination with Radioastron and SpectrXG, it is part of the Russian Spectrum Series. All observatories of this series use the Russian NAVIGATOR platform as satellite bus. The launch of WSO-UV is scheduled for 2016 and the minimum mission time is 5 years.

The observing time will be divided into a Core Program (CP), a Funding Bodies Program (FP), and Open Time (OT). The main scientific goals of the $\mathrm{CP}$ will be the study of galaxy formation to determine the diffuse baryonic content in the Universe and its chemical evolution, physics of accretion and outflow, and extrasolar planetary atmospheres and astrochemistry in the presence of strong UV radiation fields. ${ }^{1}$

Send correspondence to Sebastian Diebold

E-mail: diebold@astro.uni-tuebingen.de, Telephone: +49 70712978609

Space Telescopes and Instrumentation 2012: Ultraviolet to Gamma Ray,

edited by Tadayuki Takahashi, Stephen S. Murray, Jan-Willem A. den Herder,

Proc. of SPIE Vol. 8443, 84432X - (c) 2012 SPIE · CCC code: 0277-786/12/\$18 - doi: 10.1117/12.925967

Proc. of SPIE Vol. $844384432 X-1$ 


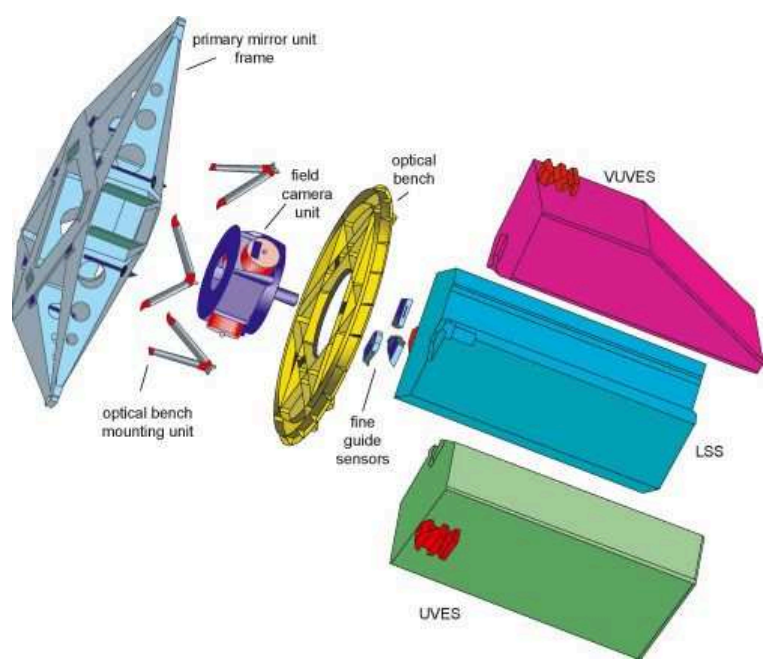

Figure 1. Sketch of the WSO-UV instrument compartment (early Phase-B-Study design)

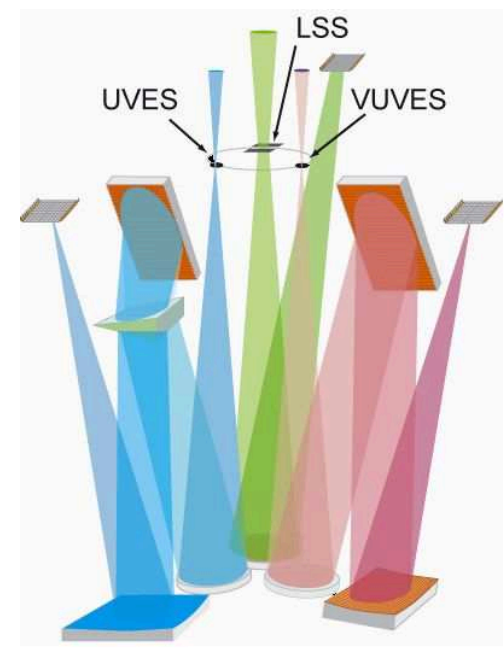

Figure 2. Principle of the High Resolution Double Echelle Spectrograph (HIRDES)

\subsection{Instrumentation on WSO-UV}

WSO-UV possesses a $1.7 \mathrm{~m}$ Ritchey-Chretien telescope with $17 \mathrm{~m}$ focal length and a field of view of $30^{\prime}$. The required pointing stability of about $0.1^{\prime \prime}$ will be achieved with three fine guidance sensors.

The main focus of the mission is on spectroscopy. Therefore, three spectrographs are mounted in the instrument compartment between mirror and satellite bus (cf. Fig. 1): Two high-resolution echelle spectrographs with a resolution $R \approx 50000$, VUVES for the VUV band $(102-176 \mathrm{~nm})$ and UVES for the UV band $(174-310 \mathrm{~nm})$, along with a low-resolution Long Slit Spectrograph (LSS, $R \approx 1000,102-310 \mathrm{~nm}$ ). The echelle spectrographs were developed in a collaboration between IAAT and ISAS Institute for Analytical Science, Berlin, under the name HIRDES (cf. Fig. 2). They are specifically designed to be equipped with microchannel plate (MCP) detectors. In addition to the spectrographs, an imaging instrument, the Imaging and Slitless Spectroscopy Instrument for Surveys (ISSIS), is placed in the focal plane of the telescope. ${ }^{2}$

\subsection{Detector Requirements}

Both HIRDES-spectrographs (VUVES and UVES) utilize two redundant MCP detectors. The LSS is drafted for the use of two MCP detectors, one for the VUV and one for the UV wavelength range.

The size of the echelle spectra at the detector surface is $30 \mathrm{~mm}$ in main dispersion direction by $40 \mathrm{~mm}$ in cross dispersion direction. Therefore, the active area for a circular detector needs to be $50 \mathrm{~mm}$ in diameter. The minimal required spatial resolution is $30 \mu \mathrm{m}$ in main dispersion direction to distribute one optical resolution element on three electronic pixels. Nevertheless, the goal is to achieve $15 \mu \mathrm{m}$. In this case, the spectral resolution is only limited by the optics. In cross dispersion direction the resolution has to be in the same order of magnitude, but it is less critical.

Both echelle spectrographs are by design very efficient, since they have a minimal number of optical elements and use high reflective coatings. Therefore, the detectors must feature a high quantum efficiency to obtain spectra of faint stars in an acceptable time frame. A critical issue in this context is solar blindness of the detectors, otherwise the background caused by straylight is defining the lower limit of the count rate, and sets a lower threshold for the faintness of possible sources. Furthermore, single photon detection is required to compensate for the jitter of the spacecraft during a long time exposure. A slit monitor records the precise position of the observed object in the slit. This information combined with a timestamp for each event gives the possibility to rearrange the photons afterwards and correct the smearing effect.

The detectors have to be capable of handling a maximal count rate of $3 \cdot 10^{5} \mathrm{~s}^{-1}$. This requirement rather aims at a short detector dead time, than at observing bright objects which will induce such high rates. In the Phase-B-Study design, a neutral density filter has been foreseen for the observation of bright sources. 


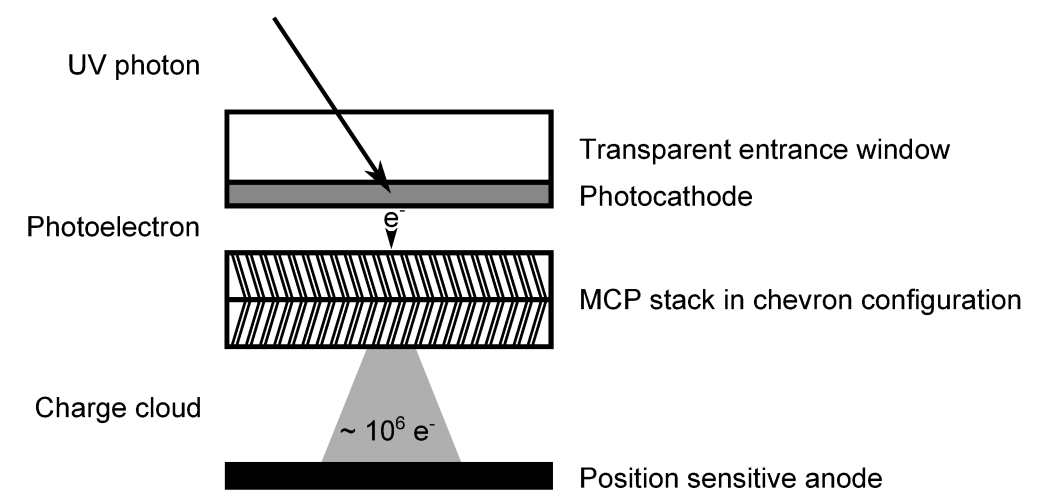

Figure 3. Principle of operation of a MCP detector with a semitransparent photocathode

There are two additional requirements which come into conflict with the ones named before: A long detector lifetime and a low power dissipation. A lifetime of at least 5 years, more likely 10 years, is necessary for achieving the planned mission lifetime. For a constant count rate, the degradation rate of MCPs is depending on the gain. From this it follows that the detector design has to ensure that the needed gain to achieve the required resolution has to be as low as possible.

The power dissipation of a single detector including the front-end electronics is limited to $8-10 \mathrm{~W}$. This value arises from the limit which was set on thermal distortions of the spectrograph structure.

\section{MCP DETECTOR PRINCIPLE}

The basic principle of a MCP detector is rather simple. An incoming photon is absorbed in a photocathode or in a bare MCP and produces a photoelectron. This electron is then accelerated towards a stack of two (Chevron stack) or three (Z stack) MCPs by an electric field. The MCPs work as electron multipliers and produce a charge cloud. This charge cloud creates a signal in a position sensitive anode, and therewith the position of the incoming photon can be reconstructed. The different detector parts mentioned here are explained in more detail in the following.

\subsection{Photocathode}

Since bare MCPs emit photoelectrons when illuminated with a UV light source, a photocathode is not crucial for the function of a MCP detector for the UV wavelength range but the quantum efficiency (QE) would be in the sub percent range. A photocathode can enhance the QE to almost $100 \%$, depending on the combination of material and photon wavelength.

Two different photocathode modes are possible. For the opaque mode, the photocathode is deposited directly on the surface of the first MCP. A wire grid with large opening ratio is placed in front of the photocathode and set on a negative potential to increase the QE by pushing back photoelectrons, which are created between the channel openings. Detectors of this type are referred to as open-face detectors. As most of the commonly used photocathodes degrade more or less rapidly under atmospheric conditions, they have to be kept in vacuum, and the detector needs a shutter mechanism if exposed to air.

The second possible mode is the so-called semitransparent mode. Thereby the detector body is either a evacuated sealed tube or actively pumped. The photocathode is deposited on the inside of a entrance window, which is in close proximity to the MCP stack. The material of the entrance window limits the wavelength range of the photons that can reach the photcathode. Compared to the opaque mode, the thickness of the photocathode is a critical parameter for the QE. On the one hand it has to be thick enough to absorb the photons, but on the other hand the escape possibility for the photoelectron has to be high. The optimal thickness can be figured out empirically. The principle of a MCP detector with a semitransparent photocathode is shown in Figure 3. 


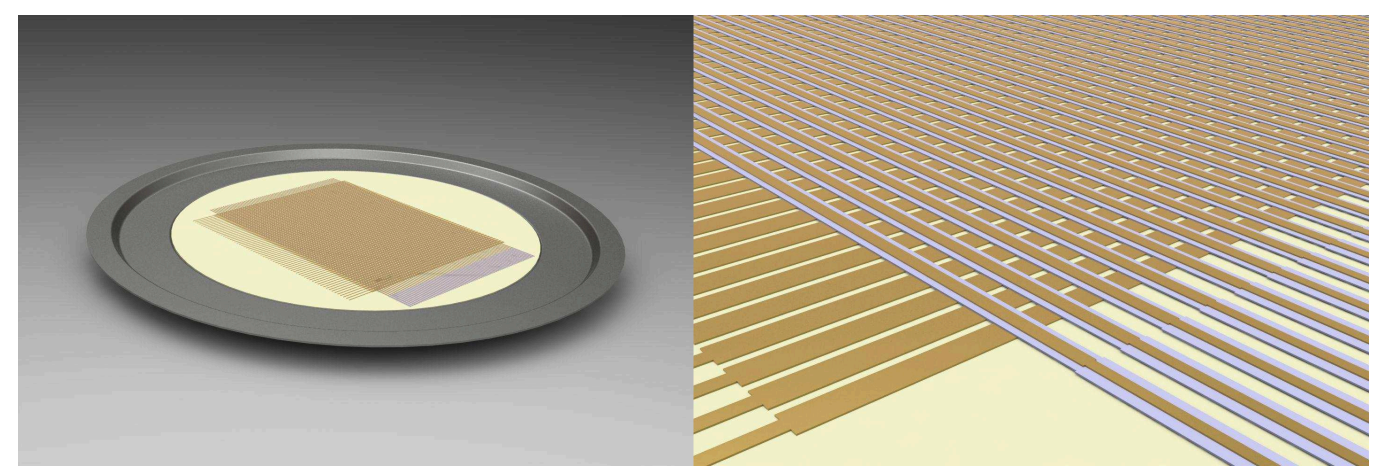

Figure 4. CAD model of the XS anode. Left: Overall view. Right: Detailed view. Top, bottom and isolation layers can be distinguished.

\subsection{Microchannel Plate}

MCPs are glass plates, typically $0.5-1 \mathrm{~mm}$ thick, with straight microscopic channels. The diameter of these channels ranges for different kinds of MCPs from some ten micrometers down to $2 \mu \mathrm{m}$. The channel surfaces are coated with a high resistive material. If a high voltage of about $1000 \mathrm{~V}$ per MCP is applied, the channels work as electron multipliers and produce a charge avalanche when hit by (photo-)electrons, ions, or neutrons. Typical gain for a single MCP is $10^{3}-10^{5}$, depending on the applied voltage. The channels are tilted with respect to the surface normal to suppress ion feedback from ionized residual gas. MCPs can only be operated in vacuum $\left(\leq 10^{-5} \mathrm{mbar}\right)$.

The lifetime (the total number of counts) of MCPs is limited. There is a maximum charge per unit area that can extracted from a MCP until the gain starts to drop. A small gain drop can be compensated by increasing the voltage but nevertheless the lifetime can only be extended efficiently by reducing the needed gain.

\subsection{Position Sensitive Anode}

A large variety of different position sensitive anodes could be used for MCP detectors. The types most widely used are wedge and strip anode, delay line anode (state of the art for the application on astronomical satellite missions), and cross strip anode (XS anode, state of the art for laboratory experiments).

In order to reduce the needed gain of the MCPs, and therefore extend the detector lifetime, we develop lowpower front-end electronics for the first application of a XS anode on board of a satellite. A detailed description of the front-end electronics can be found in Reference 3. A CAD drawing of our XS anode design is shown in Figure 4. 64 horizontal and 64 vertical electrodes will cover $44 \mathrm{~mm}$ by $33 \mathrm{~mm}$. This is slightly larger than the needed sensitive area of $40 \mathrm{~mm}$ by $30 \mathrm{~mm}$ to avoid edge effects.

\section{GALLIUM NITRIDE PHOTOCATHODE}

Reaching the lower wavelength limit of $102 \mathrm{~nm}$ for the VUV band is only possible with an open-face detector because of absorption in the window of a sealed tube detector. Therefore, we plan to use a cesium iodide (CsI) photocathode in opaque mode for this band. This is state of the art but the quantum efficiency (QE) will only be moderate, whereas a cesium-activated gallium nitride $(\mathrm{GaN})$ photocathode could reach a significantly higher QE. ${ }^{4}$ We estimate an improvement of a factor of 2 for the wavelengths around $100 \mathrm{~nm}$ and up to a factor of 10 for the wavelengths around $180 \mathrm{~nm}$. Unfortunately it is still not possible to deposit GaN directly on glass MCPs because the needed substrate temperature to grow high quality GaN films exceeds $350^{\circ} \mathrm{C}$ and would damage the MCPs.

A possible workaround is the use of $\mathrm{GaN}$ in semitransparent mode on a magnesium diflouride $\left(\mathrm{MgF}_{2}\right)$ window. The lower transmission threshold of $\mathrm{MgF}_{2}$ is about $110 \mathrm{~nm}$. This means that some nanometers at the high energy end of the VUV band will be lost, but the increase in QE will trade this off. A collaboration with the Karlsuhe 


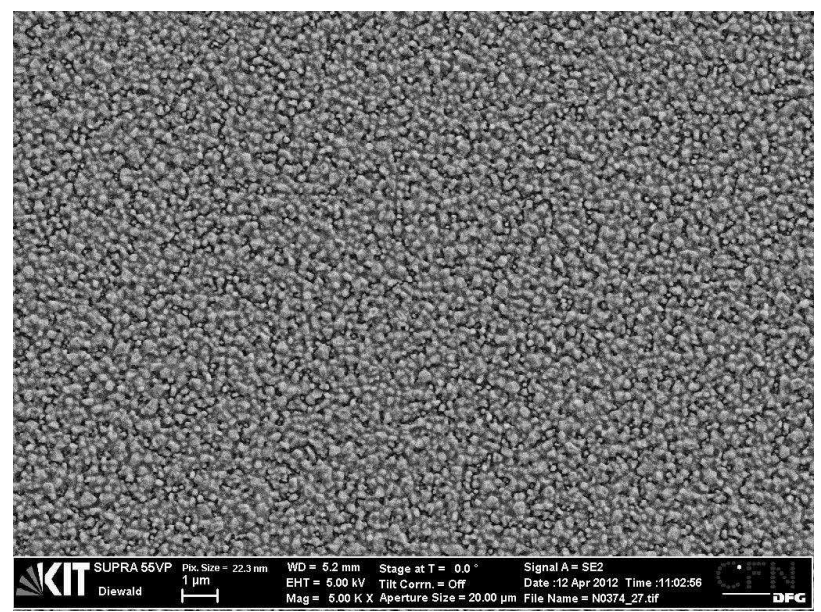

Figure 5. SEM of a GaN film (undoped) on a $\mathrm{MgF}_{2}$ substrate

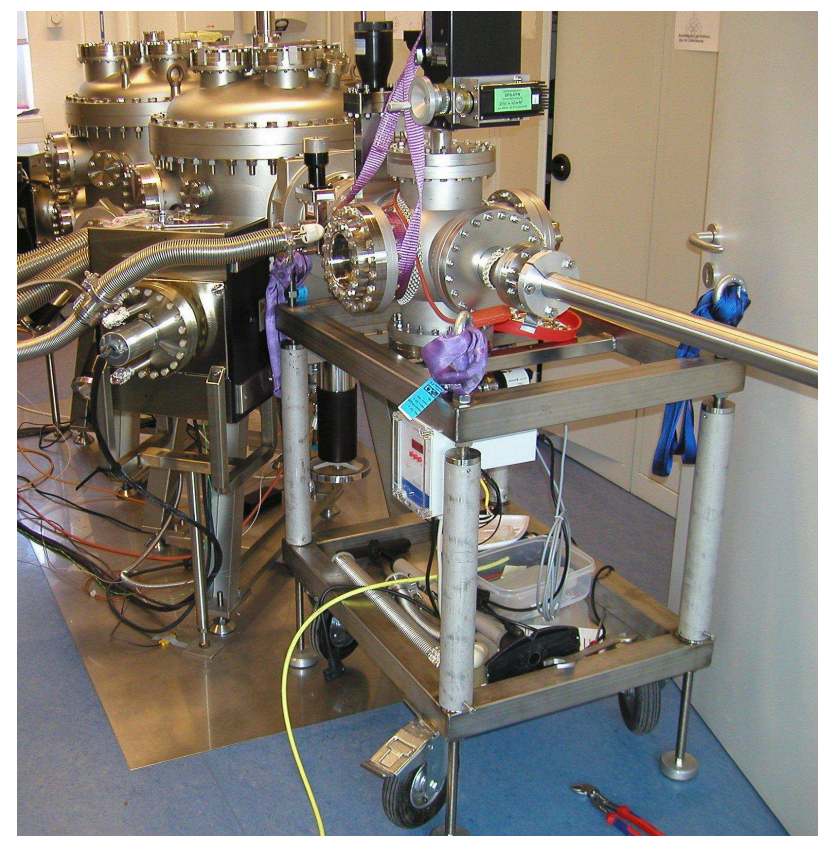

Figure 6. High vacuum transport box for the shipment of unactivated GaN samples from Karlsruhe to Tübingen

Institute of Technology (KIT) has been formed to study the deposition of magnesium doped $\mathrm{GaN}_{\text {on }} \mathrm{MgF} \mathrm{F}_{2}$. $\mathrm{A}$ scanning electron microscopy (SEM) picture of an undoped GaN film produced at KIT can be seen in Figure 5 .

For the UV band the use of semitransparent GaN will also lead to a higher QE compared to state of the art materials like cesium telluride $(\mathrm{CsTe})$. For this wavelength range the films can be deposited on a $\mathrm{MgF}_{2}$ or sapphire window without a reduction of the spectral range.

A difficulty with GaN is its rapid degradation under atmospheric conditions. To avoid degradation while shipping the unactivated samples from Karlsruhe to Tübingen, we developed and successfully tested a high vacuum transport box. This box can be attached to the deposition chamber at the KIT as well as to the activation and sealing facility at the IAAT. It can hold up to seven samples. The transport box attached to the ultra high vacuum (UHV) facility in Tübingen is shown in Figure 6. 


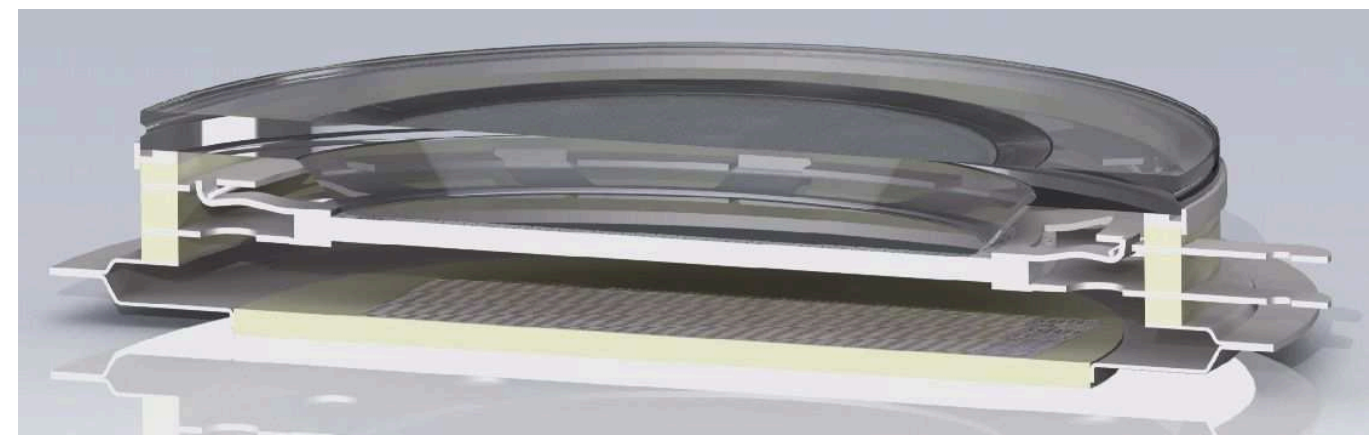

Figure 7. CAD model of our sealed tube detector design in cross-sectional view

\section{DETECTOR BODY}

A CAD model of the detector design is shown in Figure 7. To ease the production process, a circular shape of the detector tube has been chosen. The tube diameter is $8 \mathrm{~cm}$ with a $5 \mathrm{~cm}$ active area. The leak tight detector body has to provide mountings and insulated high voltage feed-throughs for the MCP stack and a leak-proof mounting for the XS anode.

It is crucial that the thermal expansion coefficients of conductive and insulating materials match closely to minimize thermal stress on the joints. We have chosen Kovar (Fe54Ni29Co17) as conductor and an aluminum oxide ceramic $\left(\mathrm{Al}_{2} \mathrm{O}_{3}\right)$ as insulator. The interfaces between these materials will be specially coated and soldered. The necessary processes are currently under development at the Empa, Dübendorf.

The design of the baseplate which holds the XS anode and the feed-throughs for the 128 anode electrodes is still pending. One possibility is to use an enlarged ceramic substrate, so that it can soldered directly to the lowest ceramic ring, another would be a baseplate flange onto which the rectangular anode is soldered. The latter is shown in Figure 7.

\section{SEALING PROCESS}

Probably the most critical step in the production of a sealed tube MCP detector is the heating of the detector body with mounted MCPs and the attachment of the entrance window with an hot indium sealing procedure. The heating of the assembled detector body to about $200^{\circ} \mathrm{C}$ is necessary to outgas the whole assembly, and, therefore, avoid a decline of the initial vacuum. Additionally a mechanically robust micro getter could be placed inside the detector tube to improve the vacuum stability.

We have set up a production facility in Tübingen consisting of two connected UHV vessels (cf. Fig. 8). In the right vessel, to which the transfer box for the GaN coated entrance windows could be connected (cf. Sec. 3), we deposit CsI and CsTe photocathodes and activate the GaN photocathodes. This vessel is equipped with quartz windows and a monochromatic UV light source and detector system to check the QE of the photocathodes already inside the vacuum vessel. The activated photocathodes are then transfered to the left vessel where the heating and sealing of the detector tubes takes place.

In order to seal the entrance window on top of the completely assembled detector body with the MCPs we will use a hot indium sealing technique. The indium is placed in a small groove on the top Kovar ring of the detector tube and heated to about $160^{\circ} \mathrm{C}$. Then the window is placed on top of the tube and fixed by applying a small contact pressure. To ensure that the indium wets the window well and a proper electrical connection is formed, we coat the contact region of the inside of the entrance window with a thin nickel chrome (NiCr) layer. The sealing process is executed under UHV conditions $\left(\approx 10^{-10} \mathrm{mbar}\right)$.

A widely known problem with the indium sealing technique is the formation of an oxide layer on the indium prior to the sealing process. This oxide layer grows after exposing the indium to air within minutes to a thickness of about $100 \mathrm{~nm}$. Afterwards this layer shields the indium bulk from further oxidation. Heating the indium to a temperature above $250^{\circ} \mathrm{C}$ under vacuum conditions reduces the indium, but for our application this heating 


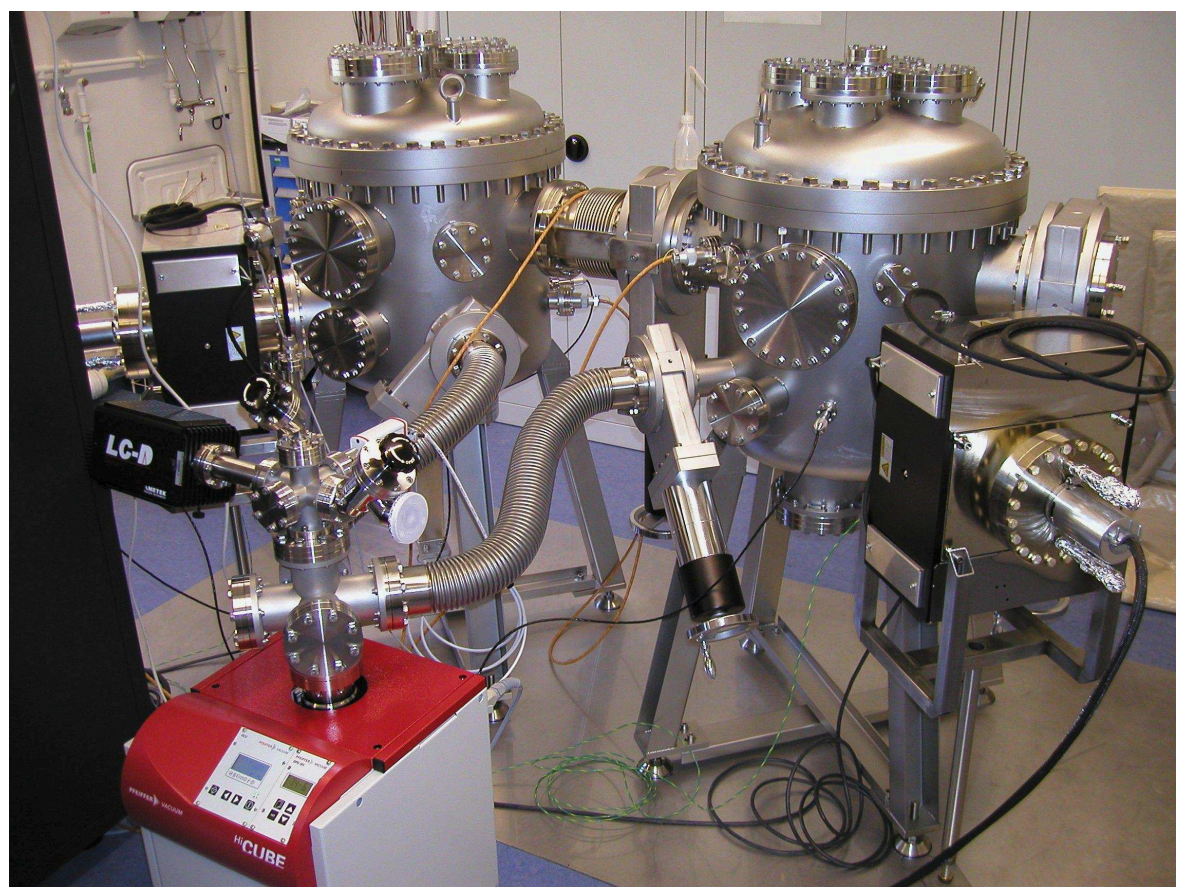

Figure 8. UHV facility at IAAT, consisting of two UHV vessels for deposition and activation of photocathodes (right vessel) and indium sealing (left vessel)

process could damage the XS anode interfaces or the anode itself. Therefore, we study the use of a hydrogen source in order to remove the oxide layer in the sealing vessel.

\section{STATUS AND OUTLOOK}

The vacuum vessels of the production facility at the IAAT have been set up and we reach the $10^{-11}$ mbar pressure regime. The interior of the vessel for the production and activation of photocathodes is almost finished and we deposited our first CsTe photocathodes. In order to test the complete chain of production processes we will use these photocathodes to produce photodiodes, which are similar to the MCP detector, but smaller $(2 \mathrm{~cm}$ in diameter) and without MCPs and a position sensitive anode. Currently we set up the interior of the sealing vessel and simultaneously the manufacturing of the detector body is studied at the Empa, Dübendorf.

The deposition of GaN on $\mathrm{MgF}_{2}$ substrates makes good progress and the KIT group started to dope the GaN with Mg. A test of the transfer box for the shipping of unactivated photocathodes from Karlsruhe to Tübingen was successful and without any difficulty.

Currently we work out the details of the transfer process of activated cathodes into the sealing vessel, the heating of the detector body and the mechanics for the sealing.

\section{ACKNOWLEDGMENTS}

This work is funded by the Bundesministerium für Wirtschaft und Technologie through the Deutsches Zentrum für Luft- und Raumfahrt e.V. (DLR) under the grant number FKZ 50 QT 1003. 


\section{REFERENCES}

[1] Werner, K., Shustov, B., Sachkov, M., Gómez de Castro, A. I., Huang, M., Kappelmann, N., and Zhao, G., "WSO-UV - Ultraviolet Mission for the Next Decade," AIP Conference Proceedings 1135, 314-317 (2009).

[2] Werner, K., Barnstedt, J., Gringel, W., Kappelmann, N., Becker-Roß, H., Florek, S., Graue, R., Kampf, D., Reutlinger, A., Neumann, C., Shustov, B., Moisheev, A., and Skripunov, E., "HIRDES - The High-Resolution Double-Echelle Spectrograph for the World Space Observatory Ultraviolet (WSO/UV)," Advances in Space Research 41, 1992-1997 (2008).

[3] Pfeifer, M., Barnstedt, J., Bauer, C., Diebold, S., Hermanutz, S., Kalkuhl, C., Kappelmann, N., Löchner, S., Schanz, T., Schmelling, M., and Werner, K., "Low-power readout electronics for microchannel plate detectors with cross strip anodes," Proceedings of SPIE 8443 (2012).

[4] Siegmund, O. H. W., Tremsin, A. S., Vallerga, J. V., McPhate, J. B., Hull, J. S., Malloy, J., and Dabiran, A. M., "Gallium nitride photocathode development for imaging detectors," in [Society of Photo-Optical Instrumentation Engineers (SPIE) Conference Series], Presented at the Society of Photo-Optical Instrumentation Engineers (SPIE) Conference 7021 (Aug. 2008). 